\title{
A single gene causes both male sterility and segregation distortion in Drosophila hybrids
}

\author{
Nitin Phadnis ${ }^{*}, 1$ and $\mathbf{H}$. Allen Orr ${ }^{1}$ \\ 1Department of Biology, University of Rochester, Rochester, NY 14627-0211, U.S.A.
}

\begin{abstract}
A central goal of evolutionary biology is to identify the genes and evolutionary forces that cause speciation, the emergence of reproductive isolation between populations. Despite the identification of several genes that cause hybrid sterility or inviability — many of which have evolved rapidly under positive Darwinian selection - little is known about the ecological or genomic forces that drive the evolution of postzygotic isolation. Here we show that the same gene, Overdrive, causes both male sterility and segregation distortion in $\mathrm{F}_{1}$ hybrids between the Bogota and USA subspecies of Drosophila pseudoobscura. This segregation distorter gene is essential for hybrid sterility, a strong reproductive barrier between these young taxa. Our results suggest that genetic conflict may be an important evolutionary force in speciation.
\end{abstract}

\begin{abstract}
Segregation distorters (also called transmission ratio distorters) are selfish genetic elements that manipulate Mendelian transmission to their own advantage. Because they are inherited by more than $50 \%$ of functional gametes, such distorters can spread rapidly through populations. Natural selection, however, favors the suppression of distortion, especially if the distorter locus resides on a sex chromosome and biases sex ratios. Theory predicts that mutations suppressing segregation distortion will invade populations once distorter alleles are at high frequency or are fixed $(1,2)$. Bouts of distortion and suppression could occur repeatedly and often. If suppressors of distortion are less than fully dominant, segregation distortion may re-appear in $\mathrm{F}_{1}$ hybrids between species. And because segregation distorters often act by destroying gametes (3), such re-expression could result in hybrid sterility $(4,5)$. The idea that intra-genomic conflict involving segregation distorters may cause hybrid sterility is controversial (4-9). Although it can explain both the observed rapid evolution of most hybrid incompatibility genes (10-15) and the preferential sterility of the heterogametic sex [an aspect of Haldane's rule (16)], direct empirical evidence is scarce (17; but see 8$)$.
\end{abstract}

Drosophila pseudoobscura pseudoobscura and Drosophila pseudoobscura bogotana (hereafter USA and Bogota, respectively) are allopatric subspecies that diverged just 155,000-230,000 years ago (18). Crosses between Bogota females and USA males produce $F_{1}$ hybrid males that are nearly completely sterile, whereas all other $F_{1}$ hybrids are fertile (19). Hybrid sterility appears to involve a single complex genetic incompatibility in which several loci are essential for the expression of full sterility. Bogota alleles at loci on the right and left arms of the $X$ chromosome ( $X R$ and $X L$, respectively) interact with dominant USA alleles on the second and third autosomes to cause hybrid sterility (20). Because these genes are essential for hybrid sterility, they could not have evolved after the attainment of complete

\footnotetext{
*This manuscript has been accepted for publication in Science. This version has not undergone final editing. Please refer to the complete version of record at http://www.sciencemag.org/. The manuscript may not be reproduced or used in any manner that does not fall within the fair use provisions of the Copyright Act without the prior, written permission of AAAS.

*Corresponding author, e-mail: pdns@mail.rochester.edu, Tel: 206-914-7270; Fax: 206-667-6497
} 
reproductive isolation. These genes may, therefore, be important components of ongoing speciation.

When aged, $F_{1}$ hybrid males (previously thought to be completely sterile) become weakly fertile. Surprisingly, these hybrid males produce progeny that are almost all daughters (21). This sex-ratio distortion is not caused by hybrid inviability but by an overrepresentation of $X$ bearing sperm among functional gametes; consequently, $X$-bearing sperm from $\mathrm{F}_{1}$ hybrid males fertilize more eggs than do $Y$-bearing sperm (21). The precise mechanism of segregation distortion may involve true meiotic drive (which acts during meiosis) or gamete killing or inactivation (which acts after meiosis); in either case, the $X$ chromosome carrying this segregation distorter enjoys a selective advantage.

Male sterility and segregation distortion in Bogota-USA hybrids both map to the same chromosomal regions and these regions show a similar pattern of complex epistasis for both phenotypes $(20,21)$. A region on the Bogota $X R$ that is tightly linked to the visible mutation sepia (se, $X R$-156.6) was identified as playing a large and essential role in both hybrid male sterility and hybrid segregation distortion. We tested if the same gene(s) cause hybrid sterility and hybrid segregation distortion by dissecting genetically the sepia region.

We generated 175 independent introgression lines in which the USA sepia region was moved into an otherwise pure Bogota background by backcrossing to the Bogota subspecies for 28 generations (14 of which involved recombination; Fig. 1A). The resulting introgression lines have genomes that derive almost entirely from Bogota except for a small chromosomal region near sepia. We tested each introgression line for hybrid sterility and segregation distortion by crossing females from these introgression lines to USA males. The resulting hybrid males should be genetically identical to $F_{1}$ hybrid males except for the small chromosomal region introgressed from USA. All 175 lines yielded hybrid males that were both fertile and produced normal ( 50:50) sex ratios (Figs 1B, S1, S2). This suggests that hybrid sterility and hybrid segregation distortion are caused either by tightly linked genes or by the same gene(s) in the region.

We genotyped all introgression lines using 41 microsatellite and 44 SNP-based diagnostic markers (SOM text). The introgression breakpoints defined a region around sepia that harbors the hybrid sterility and hybrid segregation distortion gene(s). To isolate recombinants between the sepia locus and the hybrid sterility locus (recombinants that we did not recover above), we crossed Bogota females heterozygous for an introgression to USA males and tested $s e^{+}$males for their ability to produce progeny. We found one such fertile $s e^{+}$male. Analysis of flies derived from this recombinant showed that such males produce $\sim$ 50:50 male:female progeny (Fig. S3). Genotyping revealed that the gene(s) causing hybrid sterility and hybrid segregation distortion are located to the left of sepia. Together, the introgression and fertile $s e^{+}$recombinant lines (which provided left and right breakpoints, respectively) localized the gene(s) causing hybrid sterility and hybrid segregation distortion to a $\sim 20 \mathrm{~kb}$ region that contains five predicted genes (Fig. 1C).

No functional data are available from Drosophila melanogaster for the homologsof these genes. We sequenced the entire $20 \mathrm{~kb}$ region from the Bogota subspecies and compared it to the homologous USA sequence from the D. pseudoobscura (USA) genome (22). We found no duplication, deletion, or obvious rearrangement differences between the subspecies in this region. But one of the predicted genes in the region, GA19777, shows eight non-synonymous changes, which is surprising given its small coding region (591 bp, excluding one $74 \mathrm{bp}$ intron, Table S1). Given that genes causing reproductive isolation often evolve rapidly, GA19777, which contains a DNA-binding motif, represented our best candidate for the cause of hybrid sterility and/or hybrid segregation distortion. 
To determine whether GA19777 causes these hybrid phenotypes, we first attempted to rescue the fertility of $\mathrm{F}_{1}$ hybrid males with a transgenic copy of the (fertile) USA allele of GA19777 (hereafter GA19777USA). We cloned GA19777USA along with $\sim 0.2 \mathrm{~kb}$ of upstream and $\sim 1.2 \mathrm{~kb}$ of downstream sequence into a CaSpeR-4 P-element vector. The plasmid $P\left\{w^{+}\right.$;

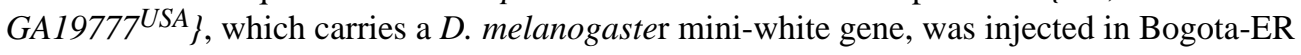
white embryos and transformants were identified by wild-type eye color. When crossed to USA white males, Bogota females heterozygous for $P\left\{w^{+}\right.$; GA19777USA $\}$produced two types of hybrid sons: those that inherited a transgenic GA19777USA along with the endogenous $G A 19777^{B O G}$ (genotype: $G A 19777^{B O G} / Y ; G A 19777^{U S A}$ ) and those that inherited only the endogenous $G A 19777^{B O G}$ allele (genotype: $G A 19777^{B O G} / Y$ ). The strength of fertility rescue in these experiments, if any, will thus depend on dominance relations between $G A 19777^{B O G}$ and the GA19777 $U S A$ transgene. Sperm motility assays showed that hybrid males that inherited the GA19777USA transgene were more fertile than those that inherited only the endogenous $G A 19777^{B O G}$ regardless of the transgenic line studied (Fig. 2). We also crossed males to Bogota white females and found that hybrid males that inherited the GA19777USA transgene produced progeny significantly more often than males that inherited only the endogenous

$G A 19777^{B O G}$, although fertility rescue was weak (Table 1). On the basis of these results, we conclude that GA19777 plays a role in hybrid male sterility.

Surprisingly, GA19777USA transgenic hybrids still produced almost all daughters (Table 1). GA19777USA transgenes do not, therefore, suppress segregation distortion. This result can be explained in at least two ways: GA19777 might cause hybrid sterility but not hybrid segregation distortion, or GA19777 might cause both hybrid sterility and hybrid segregation distortion but each phenotype has different penetrance or degree of dominance (e.g., with $G A 19777^{B O G}$ having more dominant effects on hybrid segregation distortion and more recessive effects on hybrid sterility).

To distinguish these possibilities, we performed a second transgenic experiment. In particular, we tested whether transgenic $G A 19777^{B O G}$ can induce segregation distortion. To do so, we cloned $P\left\{w^{+} ; G A 19777^{B O G}\right\}$ to the same length and coordinates as $P\left\{w^{+} ; G A 19777^{U S A}\right\}$ and transformed USA and Bogota white strains with $P\left\{w^{+} ; G A 19777^{B O G}\right\}$. As expected, no sterility or segregation distortion appeared in our transformed pure subspecies strains as such individuals lack the partner genes required for the hybrid incompatibility.

We tested whether $P\left\{w^{+} ; G A 19777^{B O G}\right\}$ can cause segregation distortion in hybrid sons produced by crossing introgression females to USA males. These sons carry all partner loci necessary for the appearance of hybrid problems (Bogota XL and USA autosomes) except for the Bogota factor(s) linked to sepia and are, therefore, fertile and non-distorting. We used females from a single introgression line (line104), which carries the smallest USA introgression near sepia. When crossed to USA males carrying $P\left\{w^{+} ; G A 19777^{B O G}\right\}$, introgression females produced two types of hybrid sons: those that inherited a transgenic GA19777BOG along with the endogenous GA19777USA (genotype: GA19777USA $/ Y ; G A 19777^{B O G}$ ) and those that inherited only endogenous GA19777USA (genotype: GA19777USA/Y) (Fig. 3). Hybrid males that inherited the $G A 19777^{B O G}$ transgene produced a strikingly female-biased sex ratio when crossed to Bogota white females, whereas control males that inherited only endogenous GA19777USA produced normal ( 50:50) sex ratios (Figure 3). Thus, GA19777 plays a role in hybrid segregation distortion.

GA19777 is predicted to encode a polypeptide with a single MADF DNA-binding domain near its C-terminus end. RT-PCR revealed that GA19777 is expressed in the testes of both pure subspecies males and in sterile $\mathrm{F}_{1}$ hybrid males (Fig. S4). The constructs from the transgene experiments caused sex-chromosome segregation distortion and rescue of fertility even when located on autosomes, showing that transgenes have an effect in trans with respect to the sex 
chromosomes (Table S2). GA19777 appears, therefore, to act through its encoded product, not through cis chromosome effects. Further controls (Fig. S5) showed that the segregation distortion/sterility effects of transgenes are not an artifact of gene dosage, but depend on the species identity of the transgene.

The segregation distortion effect of $G A 19777^{B O G}$ appears to reflect single nucleotide substitutions that have occurred since the Bogota and USA subspecies split. In contrast, other known distorter genes involve gene duplications (23-25). The segregation distortion caused by GA19777 is suppressed in pure Bogota individuals not because such individuals carry an insensitive $Y$ chromosome [the Bogota $Y$ can in fact be driven (21)], but because they are homozygous for recessive autosomal suppressors (21). Given its role in both segregation distortion (meiotic drive) and hybrid sterility, we named GA19777 Overdrive (Ovd).

To study $O v d$ 's evolutionary history, we sequenced nine additional lines of Bogota and 16 of USA and found that seven of the eight non-synonymous differences initially identified are fixed in the sample, while the remaining one (at position 61) is polymorphic within Bogota. Because Bogota strains lacking this segregating difference produce hybrids that are mostly sterile with segregation distortion (21), the change at position 61 does not seem essential for hybrid sterility or segregation distortion. We tested if GA19777 experienced recurrent positive selection by performing a McDonald-Kreitman test (26); we also tested if GA19777 experienced a recent selective sweep by calculating Tajima's $D$ (27). These tests showed no significant departures from the null hypothesis of neutrality (SOM text). This result may reflect a lack of power due to unusually low polymorphism in both subspecies (SOM text), little divergence between these taxa, or (possibly) neutral evolution at $O v d$.

Surprisingly, out-group analysis with D. miranda and D. persimilis showed that all seven nonsynonymous changes and five synonymous changes fixed between Bogota and USA occurred in the Bogota lineage. Further analysis showed that rates of non-synonymous and synonymous evolution are accelerated in the Bogota lineage relative to those expected given the species tree $\left(P=7.7 \times 10^{-6}\right.$ for non-synonymous, $P=0.0007$ for synonymous, SOM text $)$. A test for accelerated evolution $\left(K_{a} / K_{S}\right)$ in the Bogota lineage using PAML was, however, non-significant $(P=0.89$, Table S3), likely reflecting accelerated evolution at both non-synonymous and synonymous sites in Bogota.

Our results show that the same gene, $O v d$, affects both $\mathrm{F}_{1}$ segregation distortion and hybrid sterility in evolutionarily young taxa that obey Haldane's rule. Although our results do not prove a history of segregation distortion in D. pseudoobscura, our results are consistent with and suggestive of such a history - especially given the burst of substitutions at $O v d$ in the Bogota lineage. While evolutionary biologists usually explain the evolution of reproductive isolation as a side-effect of adaptation to the ecological environment, our findings support the idea that genetic conflict, a form of adaptation to the internal genomic environment, may be an important force in the evolution of postzygotic isolation.

\section{Supplementary Material}

Refer to Web version on PubMed Central for supplementary material.

\section{REFERENCES}

1. Hartl DL. Theoretical Population Biology 1975;7:168. [PubMed: 1145501]

2. Hall DW. Evolution 2004;58:925. [PubMed: 15212373]

3. Lyttle TW. Annual Review of Genetics 1991;25:511.

4. Frank SA. Evolution 1991;45(Mar):262. 
5. Hurst LD, Pomiankowski A. Genetics 1991;128(Aug):841. [PubMed: 1916248]

6. Charlesworth B, Coyne JA, Orr HA. Genetics 1993;133(Feb):421. [PubMed: 8436280]

7. Coyne JA, Orr HA. Evolution 1993;47(Apr):685.

8. Tao Y, Hartl DL, Laurie CC. Proceedings of the National Academy of Sciences of the United States of America 2001;98(Nov):13183. [PubMed: 11687638]

9. Henikoff S, Ahmad K, Malik HS. Science 2001;293(Aug):1098. [PubMed: 11498581]

10. Barbash DA, Siino DF, Tarone AM, Roote J. Proceedings of the National Academy of Sciences of the United States of America 2003;100(Apr):5302. [PubMed: 12695567]

11. Wittbrodt J, Lammers R, Malitschek B, Ullrich A, Schartl M. Embo Journal 1992;11(Nov):4239. [PubMed: 1327761]

12. Ting CT, Tsaur SC, Wu ML, Wu CI. Science 1998;282(Nov):1501. [PubMed: 9822383]

13. Presgraves DC, Balagopalan L, Abmayr SM, Orr HA. Nature 2003;423(Jun):715. [PubMed: 12802326]

14. Masly JP, Jones CD, Noor MAF, Locke J, Orr HA. Science 2006;313(Sep):1448. [PubMed: 16960009]

15. Brideau NJ, et al. Science 2006;314(Nov):1292. [PubMed: 17124320]

16. Haldane JBS. Journal of genetics 1922;12:101.

17. Coyne, JA.; Orr, HA. Speciation. Sinauer Associates; Sunderland (MA): 2004.

18. Schaeffer SW, Miller EL. Proceedings of the National Academy of Sciences of the United States of America 1991;88(Jul):6097. [PubMed: 2068088]

19. Prakash S. Genetics 1972;72(Sep):143. [PubMed: 5073854]

20. Orr HA, Irving S. Genetics 2001;158(Jul):1089. [PubMed: 11454758]

21. Orr HA, Irving S. Genetics 2005;169:671. [PubMed: 15654115]

22. Richards S, et al. Genome Research 2005;15(Jan):1. [PubMed: 15632085]

23. Merrill C, Bayraktaroglu L, Kusano A, Ganetzky B. Science 1999;283(Mar):1742. [PubMed: 10073941]

24. Tao Y, et al. Plos Biology 2007;5(Nov):2576.

25. Montchamp-Moreau C, Ogereau D, Chaminade N, Colard A, Aulard S. Genetics 2006;174:1365. [PubMed: 16387875]

26. McDonald JH, Kreitman M. Nature 1991;351(Jun):652. [PubMed: 1904993]

27. Tajima F. Genetics 1989;123(Nov):585. [PubMed: 2513255]

28. We thank AquadroCFryJGlorRMalikHPresgravesD, and the Orr lab for discussions, D. Lambert for advice on molecular techniques, H. Malik and N. Elde for help with PAML, and S. Schaeffer for fly stocks. Financial support was provided by NIH (GM51932). All sequences are available at Genbank (accession numbers FJ349335- FJ349342 and FJ418600-FJ418631). The authors declare no conflict of interest. 

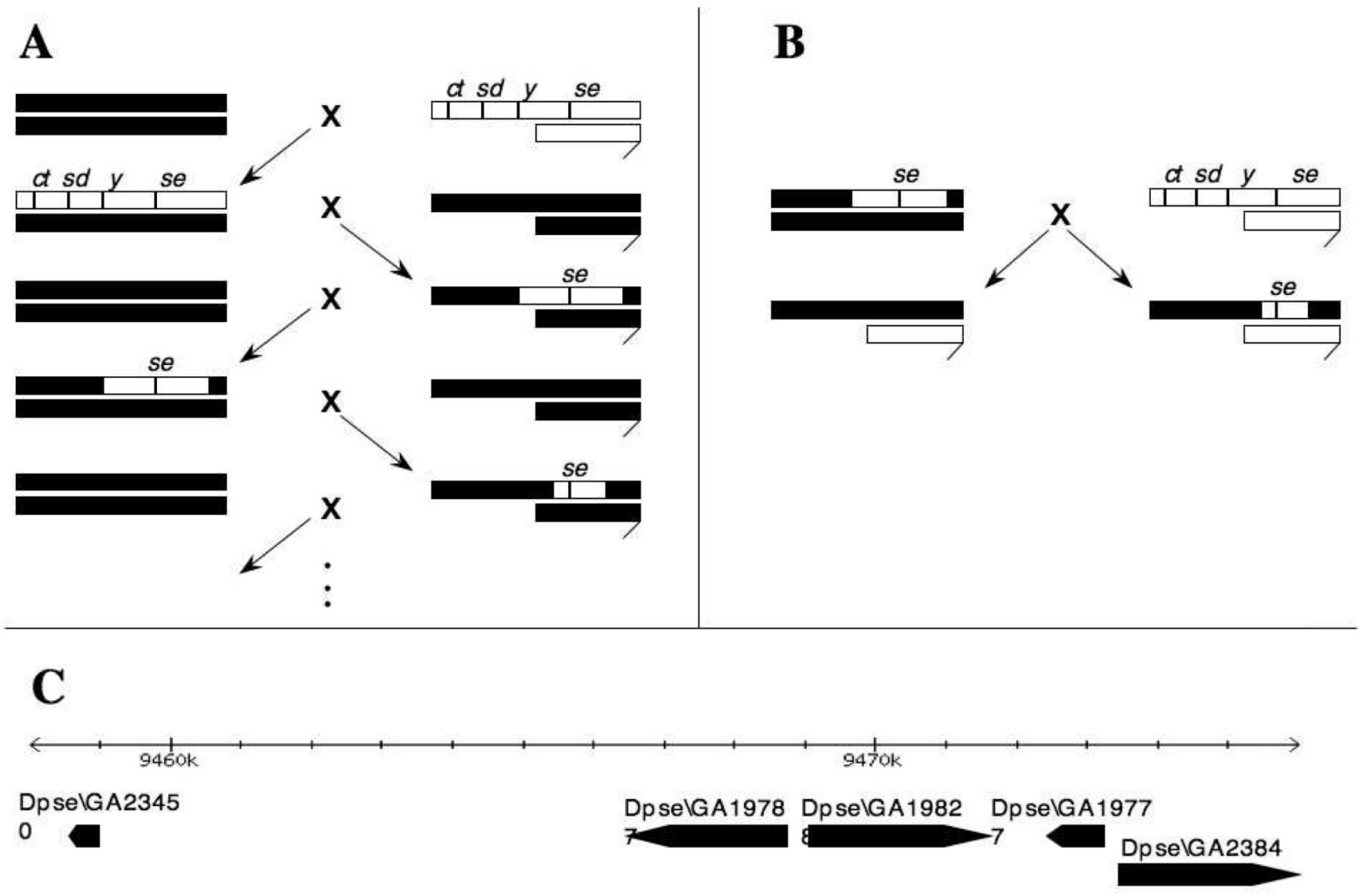

Fig.1.

A) The sepia region of the USA $X$-chromosome was introduced into an otherwise pure Bogota background using a criss-cross design: recombination occurs in females and the visible marker sepia was selected in males. Bogota material is colored black and USA material is colored white. Females have two $X$ chromosomes, males have one $X$ chromosome and one $Y$ chromosome (denoted by a hook). B) When crossed to USA males, heterozygous introgression females produce two types of hybrid males. All sepia introgression hybrid males were fertile and showed normal segregation whereas control $\mathrm{F}_{1}$ hybrid males were sterile and showed segregation distortion, as expected. C) Hybrid sterility and segregation distortion both mapped to a region spanning $\sim 20 \mathrm{~kb}$ that included five predicted genes of which GA19777 is the fastest evolving. 


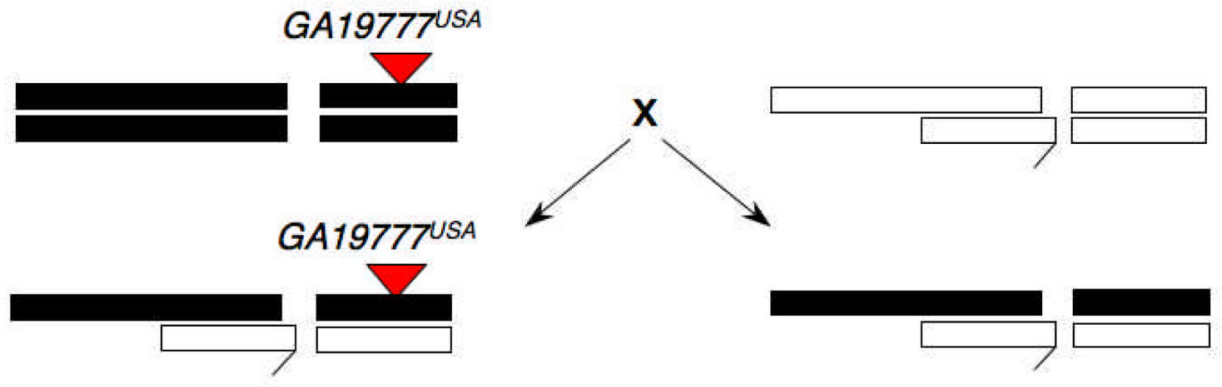

$\%$ fertile

$\begin{array}{llll}\text { USA1-1 } & 74(N=155) & 25(N=130) & P=7.18 \mathrm{E}-55 \\ \text { USA1-2 } & 91(N=113) & 24(N=111) & P=1.16 \mathrm{E}-62 \\ \text { USA1-7 } & 79(N=128) & 20(N=123) & P=4.43 \mathrm{E}-67\end{array}$

Fig. 2.

$P\left\{w^{+} ; G A 19777^{U S A}\right\}$ rescues fertility in $\mathrm{F}_{1}$ hybrid males as measured blind in a sperm motility assay. Three independent transgenic strains were tested; the total number of males tested per genotype is denoted by $N$. The two genotypes are identical except for presence/absence of the construct. $P$ values were calculated using $\chi^{2}$ tests with one degree of freedom. 

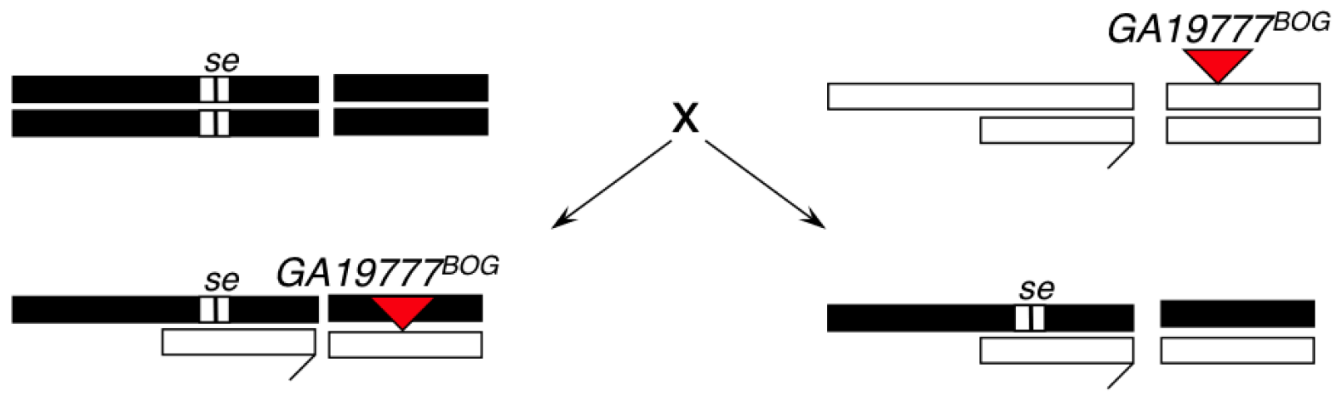

\begin{tabular}{cccc}
\hline BOG1-1 & $N=79$ & $N=90$ & \\
\hline & & & \\
\% daughters & $87.39(1.25)$ & $52.51(1.00)$ & $P=3.11 \mathrm{E}-51$ \\
Total progeny & $33.34(2.68)$ & $50.52(3.27)$ & $P=0.0001$ \\
No. of daughters & $28.37(2.27)$ & $26.32(1.75)$ & $P=0.4711$ \\
& & & \\
\hline BOG1-2 & $N=54$ & $51.29(0.98)$ & $P=1.75 \mathrm{E}-40$ \\
\hline & & $59.47(3.91)$ & $P=1.70 \mathrm{E}-09$ \\
\% daughters & $86.82(1.64)$ & $30.42(2.05)$ & $P=0.0024$ \\
\hline Notal progeny & $25.44(2.89)$ & &
\end{tabular}

Fig. 3.

$P\left\{w^{+} ; G A 19777^{B O G}\right\}$ induces segregation distortion in sepia introgression hybrid males.

Sepia introgression hybrid males that inherit the $P\left\{w^{+} ; G A 19777^{B O G}\right\}$ transgene show significantly female-biased progeny compared to males that do not inherit the transgene. The two genotypes are identical except for presence/absence of the construct. Segregation distortion is also accompanied by a reduction in the total number of progeny and, in the case of BOG1-2, in the number of daughters produced. Two independent transgenic strains (BOG1-1 and BOG1-2) were tested; $N$ denotes the number of fathers tested per genotype; mean values along with S.E. (in brackets) are reported. $P$ values were calculated using ANOVA. Transgene males were inferred from the presence of $w^{+}$individuals among $F_{1}$ and $F_{2}$ progeny. We modified the cross for the transgenic Bogota strain (BOG1-2): we first crossed introgression females to Bogota males heterozygous for $P\left\{w^{+} ; G A 19777^{B O G}\right\}$. When crossed to USA white males, these females produce two types of hybrid sons: those that inherit a transgenic GA19777BOG along with endogenous GA19777USA and those that inherit only endogenous GA19777USA. Importantly, with both BOG1-1 and BOG1-2, transgenic hybrid males produced (rare) white and wild-type sons in roughly equal numbers, showing that sex ratio distortion is not due to transgene-induced male lethality. 
Iี

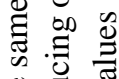

을

일

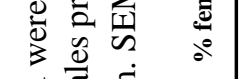

跣

品

可

के

缶

踏

豆焉

言苛

政

娄

б.

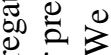

突宕

$z \quad \mathrm{w}^{0}$

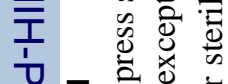

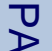

势

要

훨

은

过

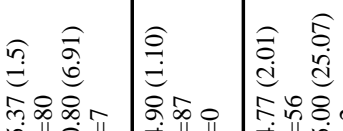

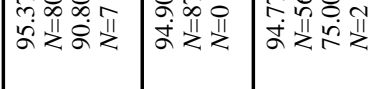

ญ

ट

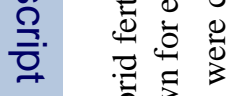

尝焉

랑

음

要

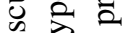

政

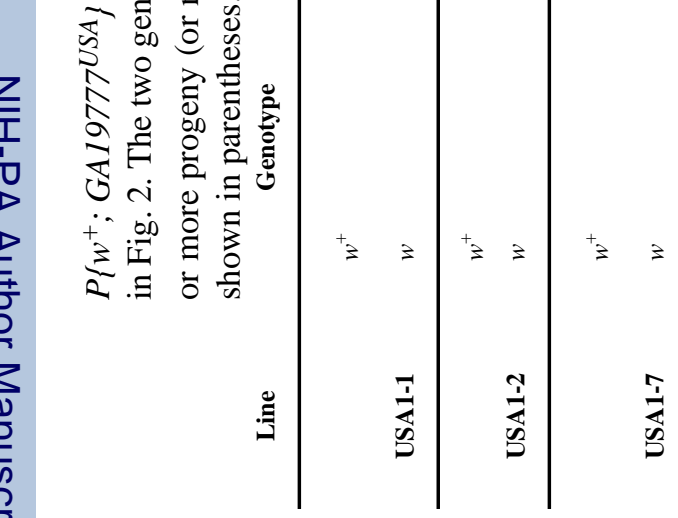

(1)

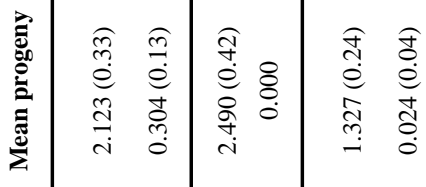

ปัต

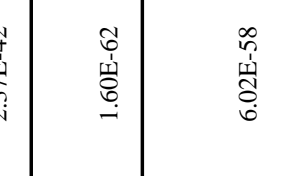

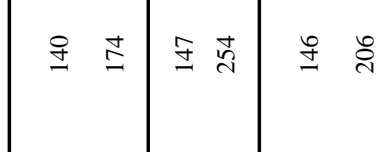

$\infty r$ so inc

훙 